\title{
CONJUGATIONS ON COMPLEX MANIFOLDS AND EQUIVARIANT HOMOTOPY OF $M U^{1}$
}

\author{
BY PETER S. LANDWEBER
}

\section{Communicated by Pierre Conner, November 1, 1967}

1. Introduction. Let $\rho: \Omega_{*}^{U} \rightarrow \mathfrak{N}_{*}$ denote the natural homomorphism from the stably complex bordism ring in to the unoriented bordism ring. Milnor showed in [8] that the image of $\rho$ consists of all squares $\left([M]_{2}\right)^{2}$ in $\mathfrak{N}_{*}$. Since $\mathfrak{N}_{*}$ is a polynomial algebra over $Z_{2}$, an epimorphism $R: \Omega_{2 n}^{U} \rightarrow \Re_{n}$ is defined by the condition that $R^{2}=\rho$. Milnor made use of the following result of Conner and Floyd [3, p. 64]: if $\tau$ is a conjugation on a closed almost complex $2 n$-manifold $M$, then the fixed point set $F(M)$ is an $n$-manifold and $[M]_{2}=\left([F(M)]_{2}\right)^{2}$ in $\mathfrak{R}_{2 n}$, i.e. $R([M])=[F(M)]_{2}$. Hence, if a conjugation is present we may regard $R$ as "passage to the fixed point set." We shall develop a bordism theory in which such a "fixed point homomorphism" is a natural feature.

From the homotopy point of view, $\Omega_{*}^{U}$ coincides with the (stable) homotopy $\pi_{*}(M U)$ of the Milnor spectrum $M U$ [7]. In fact, the Thom spaces $M U(n)$ carry involutions making it possible to define equivariant homotopy groups $\Omega_{p, q}^{U}=\pi_{p, q}(M U)$. The details follow.

Give $C^{m}$ the involution $\left(z_{1}, \cdots, z_{m}\right) \mapsto\left(\bar{z}_{1}, \cdots, \bar{z}_{m}\right)$. Then the Grassmannian $G_{n}\left(C^{m}\right)$ of $n$-planes in $C^{m}$ inherits an involution, as does the classifying space $B U(n)=G_{n}\left(C^{\infty}\right)$. Moreover, the universal complex $n$-plane bundle $E^{n} \rightarrow B U(n)$ inherits an involution which makes $E^{n}$ a real vector bundle over the real space $B U(n)$ in the sense of Atiyah [1]. Thus $M U(n)=B\left(E^{n}\right) / S\left(E^{n}\right)$ is endowed with an involution fixing the base point. Notice that the corresponding fixed point sets are $R^{m}, G_{n}\left(R^{m}\right), B O(n)$ and $M O(n)$.

Following Atiyah [1] let $B^{p, q}$ and $S^{p, q}$ denote the unit ball and unit sphere in a Euclidean space $R^{p, q}$ of dimension $p+q$ carrying an orthogonal involution with fixed point set $R^{q}$. If $X$ is a space with involution and fixed base point $*$, let $\pi_{p, q}(X)$ denote the set of equivariant homotopy classes of maps $\left(B^{p, q}, S^{p, q}\right) \rightarrow(X, *)$. For $q \geqq 2, \pi_{p, q}(X)$ is an abelian group.

There are equivariant suspension maps $i_{n}: M U(n) \wedge\left(B^{1,1} / S^{1,1}\right)$ $\rightarrow M U(n+1)$, and so homomorphisms

$$
\pi_{p+k, q+k}(M U(k)) \rightarrow \pi_{p+k+1, q+k+1}(M U(k+1)) .
$$

${ }^{1}$ This research was supported in part by National Science Foundation Grant GP-6567. 
Hence we may define

$$
\Omega_{p, q}^{U}=\pi_{p, q}(M U)=\lim _{k \rightarrow \infty} \pi_{p+k, q+k}(M U(k))
$$

for integers $p, q$. There is a forgetful homomorphism $\psi$, and a fixed point homomorphism $\phi$ obtained by restriction to the fixed point sets:

$$
\Omega_{p+q}^{U} \stackrel{\psi}{\leftarrow} \Omega_{p, q}^{U} \stackrel{\phi}{\rightarrow} \Re_{q} .
$$

We shall state a number of results about the groups $\Omega_{p, q}^{U}$ and the homomorphisms $\psi$ and $\phi$. The results of [5], on fixed point free conjugations and the existence of equivariant maps are a by-product of this study. A similar investigation of equivariant stable stems has been made by Bredon [2].

2. The exact sequence. The inclusions $R^{p+k, q+k} \rightarrow R^{p+k+1, q+k}$ give rise to a homomorphism $\chi$ so that the diagram

$$
\begin{gathered}
\Omega_{p+1, q}^{U} \stackrel{\chi}{\rightarrow} \Omega_{p q}^{U} \\
\phi \searrow \quad \measuredangle \phi \\
\Re_{q}
\end{gathered}
$$

is commutative. The image of $\chi$ consists of elements of order 2. As in [6] there is an exact sequence

$$
\cdots \rightarrow \Omega_{p+1, q}^{U} \stackrel{\chi}{\rightarrow} \Omega_{p, q}^{U} \stackrel{\psi}{\rightarrow} \Omega_{p+q}^{U} \stackrel{\omega}{\rightarrow} \Omega_{p+1, q-1}^{U} \rightarrow \cdots .
$$

It follows from the exact sequence of [6] that $\phi: \Omega_{p, q}^{U} \rightarrow \mathfrak{N}_{q}$ is an isomorphism for $p+q<0$; this gives a basis for induction on $p+q$.

THEOREM 2.2. $\Omega_{p, q}^{U}$ is a finitely generated abelian group in which all torsion is of order 2. The torsion subgroup is the kernel of $\psi: \Omega_{p, q}^{U} \rightarrow \Omega_{p+q}^{U}$.

3. Transversality. Given an equivariant map $f$ from $\left(B^{p+k, q+k}\right.$, $\left.S^{p+k, q+k}\right)$ into $(M U(k), *)$, is $f$ equivariantly homotopic to a map $g$ which is transversal to $B U(k) \subset M U(k)$ ? (As is customary, we approximate $B U(k)$ and $M U(k)-\{*\}$ by smooth manifolds.) That this is not generally true follows from the fact that $\phi: \Omega_{p, q}^{U} \rightarrow \mathfrak{N}_{q}$ is an isomorphism for $p+q<0$.

TheOREM 3.1. If $p \geqq q$, each element of $\Omega_{p, q}^{U}$ is represented by a map $f:\left(B^{p+k, q+k}, S^{p+k, q+k}\right) \rightarrow(M U(k), *)$ which is transversal to $B U(k)$ $\subset M U(k)$.

This follows by examination of a more general situation, in the 
category of smooth manifolds with involution and smooth equivariant maps. Let $f: M \rightarrow W$ be given, and let $V$ be a closed invariant submanifold of $W$. We assume that each fixed point set $F(M), F(V)$, $F(W)$ is of uniform dimension. Put $m=\operatorname{dim} M, m^{\prime}=\operatorname{dim} F(M)$, etc.

LEMMA 3.2. If $\left(m-2 m^{\prime}\right)+\left(v-2 v^{\prime}\right) \geqq\left(w-2 w^{\prime}\right), f$ is equivariantly homotopic to a map $g$ which is transversal to $V$.

Corollary 3.3. The diagram

$$
\begin{gathered}
\Omega_{n, n}^{U} \stackrel{\psi}{\rightarrow} \Omega_{2 n}^{U} \\
\phi \searrow \quad \swarrow R \\
\mathfrak{N}_{n}
\end{gathered}
$$

is commutative.

CoRollaRY 3.4. The homomorphism $\phi: \Omega_{p, q}^{U} \rightarrow \mathfrak{N}_{q}$ is onto if $p \leqq q$ and is zero if $p>q$.

The sequence

$$
0 \rightarrow \Omega_{n+1, n}^{U} \stackrel{\chi}{\rightarrow} \Omega_{n, n}^{U} \stackrel{\psi}{\rightarrow} \Omega_{2 n}^{U} \rightarrow 0
$$

is exact. I conjecture that $\Omega_{n+1, n}^{U}=0$ for all $n$, and have verified this for $n \leqq 4$.

4. The spectral sequence. We do not have a complete description of the groups $\Omega_{p, q}^{U}$. In particular, the extent of the torsion and the image of $\psi$ are not known in general. The difficulties are measured by the spectral sequence of the bigraded exact couple (2.1), which we now write as

$$
\cdots \rightarrow \Omega_{p+1, q}^{U} \stackrel{\chi}{\rightarrow} \Omega_{p, q}^{U} \stackrel{\psi}{\rightarrow} E_{p, q}^{1} \stackrel{\omega}{\rightarrow} \Omega_{p+1, q-1}^{U} \rightarrow \cdots
$$

where $E_{p, q}^{1}=\Omega_{p+q}^{U}$. The differential $d^{r}: E_{p-r, q+1}^{r} \rightarrow E_{p, q}^{r}$ of the spectral sequence $\left\{E_{p, q}^{r}\right\}(r>0)$ arises from the diagram

$$
\begin{gathered}
\Omega_{p, q}^{U} \stackrel{\psi}{\rightarrow} E_{p, q}^{1} \\
\downarrow \chi^{r-1} \\
E_{p-r, q+1}^{1} \stackrel{\omega}{\rightarrow} \Omega_{p-r+1, q}^{U} .
\end{gathered}
$$

We are able to determine $d^{1}$ and $d^{3}\left(d^{2}=0\right)$, and so reach the following conclusions. 
THEOREM 4.1. (a) If $p \not \equiv q \bmod 4, \Omega_{p, q}^{U}$ is finite; (b) if $p-q \equiv 4 \bmod 8$, $\psi: \Omega_{p, q}^{U} \rightarrow \Omega_{p+q}^{U}$ has image $2 \Omega_{p+q}^{U}$; (c) if $p \equiv q \bmod 8$, the image of $\psi$ contains $2 \Omega_{p+q}^{U}+[C P(1)] \Omega_{p+q-2}^{U}$.

CoRollaRy 4.2. If $p \equiv q \bmod 4, \Omega_{p, q}^{U}$ has the same rank as $\Omega_{p+q}^{U}$.

The differential $d^{1}: E_{p-1, q+1}^{1} \rightarrow E_{p, q}^{1}$ is zero if $p \neq \equiv q \bmod 4$, and is multiplication by 2 otherwise. This is proved with $K$-theory and $K R$-theory characteristic numbers [4], [9]; notice that the composition $\tilde{K}\left(S^{n}\right)^{r} \rightarrow[K O] \sim\left(S^{n}\right) \stackrel{c}{\rightarrow} \tilde{K}\left(S^{n}\right)$ is zero if $n \neq 0 \bmod 4$, and is multiplication by 2 otherwise. Thus $E_{p, q}^{2} \cong \Omega_{p+q}^{U} \otimes Z_{2}$ if $p \equiv q \bmod 4$, otherwise $E_{p, q}^{2}=0$. Moreover, $E^{3}=E^{2}$. With the help of characteristic numbers, we show that $d^{3}: E_{p-3, q+1}^{3} \rightarrow E_{p, q}^{3}$ is multiplication by $[C P(1)]$ if $p \equiv q$ mod 8 , otherwise $d^{3}=0$. Then $E^{7} \cong \cdots \cong E^{4} ;$ I conjecture that $d^{7}: E_{p-7, q+1}^{7} \rightarrow E_{p, q}^{7}$ is multiplication by the class of the quadric $Q^{6}$ if $p \equiv q \bmod 16$, otherwise $d^{7}$ is zero.

\section{REFERENCES}

1. M. F. Atiyah, K-theory and reality, Quart. J. Math. 17 (1966), 367-386.

2. G. E. Bredon, Equivariant stable stems, Bull. Amer. Math. Soc. 73 (1967), 269-273.

3. P. E. Conner and E. E. Floyd, Differentiable periodic maps, Springer-Verlag, Berlin, 1964.

4. - The relation of cobordism to $K$-theories, Springer Lecture Notes, Vol. 28, Springer, Berlin, 1966.

5. P. S. Landweber, Fixed point free conjugations on complex manifolds, Ann. Math. (to appear).

6. J. Levine, Spaces with involution and bundles over $P^{n}$, Amer. J. Math. 85 (1963), 516-540.

7. J. W. Milnor, On the cobordism ring and a complex analogue, Amer. J. Math. 82 (1960), 505-521.

8. - On the Stiefel-Whitney numbers of complex manifolds and of spin manifolds, Topology 3 (1965), 223-230.

9. R. E. Stong, Relations among characteristic numbers. I, Topology 4 (1965), 267-281.

UNIVERSITY OF VIRGINIA 